\title{
Identification of FBXW7 $\alpha$-regulated genes in M1-polarized macrophages in colorectal cancer by RNA sequencing
}

Yupeng Long, MD, PhD, Yujun Zhu, MD, PhD.

\begin{abstract}

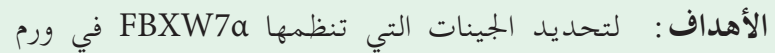
البلاعم المستقطبه في سرطان القولوّن والمستقيم.

الطريقة : أجريت هذه الدراسة التجريبية خلال الفترة بين يونيو

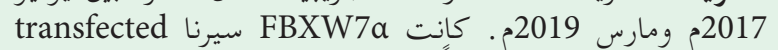

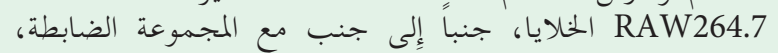

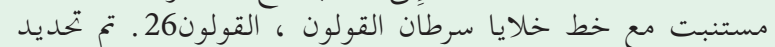

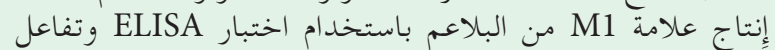

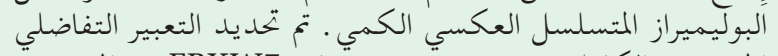

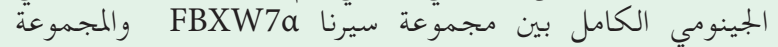

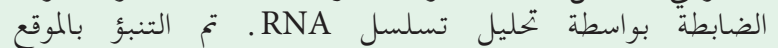

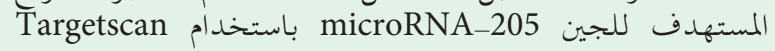
والتحقق منه بواسطة اختبار لوسيفيراز . عن طريق مئنام محاكاة

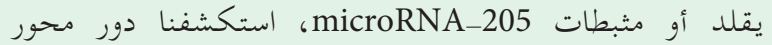
الرتطة FBXW7a / microRNA_205 في تنظيم استقطاب الخلايا الضامة

$$
\text { المرتبطة بالورم (TAM) ( T) . }
$$

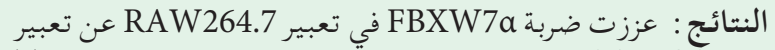
iNOS و COX-2

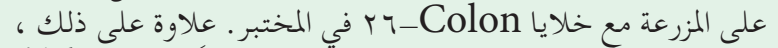

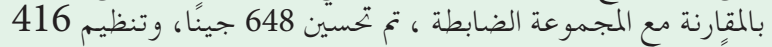

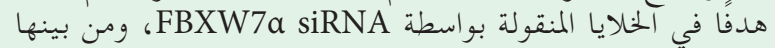

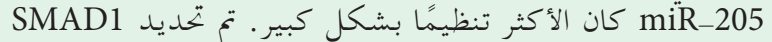

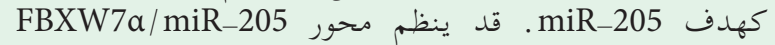
استقطاب TAM من خلال التأثير على تعبير SMAD1.

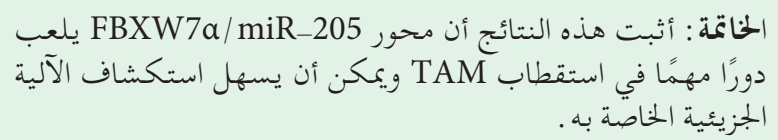

Objectives: To determine the FBXW7 $\boldsymbol{\alpha}$-regulated genes in tumor-polarized macrophages in colorectal cancer.

Methods: This experimental study was performed between June 2017 and March 2019. FBXW7 $\alpha$ siRNA transfected RAW264.7 cells, together with the control group, were co-cultured with the colon cancer cell line, Colon-26. M1 marker production from the macrophages was determined by ELISA and quantitative reverse transcription-polymerase chain reaction. Whole genomic differential expression between the FBXW7 $\alpha$ siRNA group and the control group were determined by RNA-sequencing analysis. The target site of the microRNA-205 gene was predicted using Targetscan and was verified by the luciferase assay. By transfecting mimics or inhibitors of microRNA-205, we explored the role of FBXW7 $\alpha /$ microRNA-205 axis in regulating the polarization of tumor-associated macrophages (TAM).

Results: FBXW7 $\alpha$ knockdown in RAW264.7 enhanced the expression of cyclooxigenase (COX)-2 and inducible nitric oxide synthase (iNOS), mRNA expression and IL6, IL12, p40, and tumor necrosis factor- $\alpha$ (TNF $\alpha$ ) production upon co-culture with Colon-26 cells in vitro. Further, compared with the control group, 648 genes in total were enhanced and 416 targets were downregulated in FBXW7 $\alpha$ siRNA transfected cells, among which miR205 was the most significantly upregulated. SMAD1 was identified as an miR-205 target. The FBXW7 $\alpha / \mathrm{miR}$ 205 axis might regulate TAM polarization by affecting SMAD1 expression.

Conclusion: These results prove that the FBXW7 $\alpha / m i R-205$ axis plays an important role in TAM polarization and could facilitate further exploration of its molecular mechanism.

Saudi Med J 2019; Vol. 40 (8): 766-773

doi:10.15537/smj.2019.8.24361

From the Department of Clinical Laboratory (Long), and from the Department of General Surgery (Zhu), Army 958 Hospital of the Chinese People's Liberation Army, Chongqing, China.

Received 12th March 2019. Accepted 3rd July 2019.

Address correspondence and reprint request to: Dr. Yupeng Long, Department of Clinical Laboratory, Army 958 Hospital of the Chinese People's Liberation Army, Chongqing, China. E-mail: lyp_emily@163.com ORCID ID: orcid.org/0000-0002-2219-2272 
T he mortality rate of colorectal cancer (CRC) ranks fourth among all malignant tumors. ${ }^{1}$ Colon cancer pathogenesis is generally considered a result of the genetic and epigenetic changes in colon epithelium leading to adenoma development and further progress to cancer, and this process is accompanied by changes in the composition and function of the tumor microenvironment. ${ }^{2}$

Macrophages derived from circulating monocytes are the major components of the tumor microenvironment, and are generally divided into proinflammatory polarization (M1 polarization) and anti-inflammatory polarization (M2 polarization). ${ }^{3}$ The main phenotype of tumor-associated macrophages (TAM) is M2 polarization, which can contribute to cancer development. Proinflammatory polarization macrophages play dual roles in regulating tumor development. Proinflammatory polarization macrophages can further induce carcinogenesis through prolonged secretion of pro-inflammatory mediators in a chronic inflammatory environment. However, in contrast, recent studies have shown that stimulating TAM to M1 polarization can reduce tumor size and metastasis. Considering that the colon is one of the most densely macrophage-populated organs, it is important to study the relationship between macrophages and colon cancer. ${ }^{2}$

A series of new tumor suppressor genes have been discovered and identified, including members of the FBXW7 family. ${ }^{4}$ Recent studies have shown that the FBXW7 family can regulate the occurrence, development, and metastasis of CRC. Kothari et al, ${ }^{5}$ indicated that FBXW7 gene mutation can increase the risk of CRC. The study by Xie et al, ${ }^{6}$ also reported a similar conclusion. Although increasing studies indicate that the FBXW7 family may be an important target for CRC treatment, how the FBXW7 family regulates the molecular mechanism of tumorigenesis is poorly understood. Previous reports have indicated that $\mathrm{FBXW} 7 \alpha$ may regulate inflammatory signaling in macrophages. ${ }^{7}$ Therefore, we designed this study to address whether FBXW7 $\alpha$-regulated macrophage function can mediate the development of tumors. In this study, a robust set of FBXW7 $\alpha$-regulated genes were identified by RNA sequencing analysis and we found that miR-205 was the most distinctly differentially expressed target, and then the mechanism of the

Disclosure. Authors have no conflict of interests, and the work was not supported or funded by any drug company.
FBXW7a/miR-205 axis in colon cancer development was further elucidated.

Methods. This experimental study was performed from June 2017 until March 2019. The Chinese Army 958 hospital's Ethics Committee approved this study.

We used the PubMed search engine established by the National Center for Biotechnology Information (NCBI) of the United States to search for prior related research.

Cell culture. We added 10\% fetal calf serum (FCS) (Invitrogen, Grand Island, NY, USA) and $100 \mathrm{U} / \mathrm{ml}$ streptomycin and $100 \mathrm{U} / \mathrm{ml}$ penicillin (Hyclone laboratories Inc., South UT, USA) to Dulbecco's modified Eagle medium (DMEM) to prepare the cell culture medium for Colon-26 and RAW 264.7 cells. The culture environment was 5\% carbon dioxide and $37^{\circ} \mathrm{C}$ with humidified air in an incubator (Thermo Fisher, Waltham, MA, USA).

Co-cultivation of colon cancer cells and macrophages. Colon-26 cells were inoculated into Transwell inserts (Corning Incorporated, Corning, NY, USA). RAW264.7 was inoculated into cell culture plates. After the cells were cultured for 24 hours, the Transwell inserts were placed in the cell culture plate and the cells were replenished with the culture medium, and the culture was continued for 24 hours.

Cells transfection. The exogenous plasmid was transfected into cells using Lipofectamine 2000 (Invitrogen, Grand Island, NY, USA). We constructed the siRNA vector of FBXW7 $\alpha$ and transfected it into RAW 264.7 cells to establish the macrophages with reduced expression of FBXW7 $\alpha$.

Cytokine assay. The inflammatory factors secreted by cells were detected using ELISA. The ELISA kits used here were all obtained from R\&D (Minneapolis, MN, USA). Specific experimental steps and data analysis methods were performed as per the manufacturer's protocols.

Quantitative reverse transcription-polymerase chain reaction ( $q R T-P C R$ assay). We used TRIzol reagent (Invitrogen, Grand Island, NY, USA) to obtain the total RNA from cells. Subsequently, qRT-PCR was performed using the PrimeScript RT-PCR kit (Takara, Bio Inc., Shiga, Japan) on an IQ5 fluorescence quantitative PCR detector (Bio-Rad, Hercules, CA, USA). Primer sequences for all M1 polarization markers and $\beta$-actin were obtained from previous literature. ${ }^{3}$ The following parameters were used for the reverse transcription reaction: $65^{\circ} \mathrm{C}$ for 5 minutes, $37^{\circ} \mathrm{C}$ for 15 minutes, and finally $98^{\circ} \mathrm{C}$ for 5 minutes. The following parameters were used for the subsequent PCR reaction: $95^{\circ} \mathrm{C}$ for 
30 seconds, then 40 cycles of $95^{\circ} \mathrm{C}$ for 5 seconds, and $60^{\circ} \mathrm{C}$ for 5 seconds, and finally $72^{\circ} \mathrm{C}$ for 30 seconds.

$R N A$-sequencing assay. We fragmented the mRNA of the sample into $200 \mathrm{bp}$. Subsequently, the TruSeq RNA LT/HT sample preparation kit (Illumina, San Diego, CA, USA) was used to construct the cDNA Library of the collected RNA and to synthesize the first and second strands of the collected RNA. Agilent 2200 TapeStation and Qubit 2.0 (Life Technologies, Carlsbad, CA, USA) were used to evaluate the purity of the DNA, and then the cDNA was diluted to $10 \mathrm{pM}$ and sequenced using a HiSeq instrument (Illumina, San Diego, CA, USA).

Western blotting assay. Cells were lysed to obtain the protein lysate (Pierce, Rockford, IL, USA). The supernatant of the lysate and cell components mixture was centrifuged to extract the protein. The protein quantification was performed by BCA assay (Pierce, Rockford, IL, USA). The purified protein was separated by sodium dodecyl sulfate polyacrylamide gel electrophoresis (SDS-PAGE) and transferred onto a polyvinylidene difluoride membrane. The membrane was sealed with $5 \%$ skimmed milk powder containing $0.05 \%$ Tween 20 Tris buffer saline $(\mathrm{pH} \mathrm{7.4)}$ and incubated with the primary antibody (Santa Cruz, Delaware Avenue, CA, USA) at 1:200 followed by secondary antibody (Santa Cruz, Delaware Avenue, CA, USA) at 1:5000. The target protein was detected by enhanced chemiluminescence (ECL) and film exposure.

Luciferase assay. To verify whether SMAD1 is the target of microRNA-205, we constructed a luciferase reporter gene of SMAD1 (pMIR-SMAD1-wild-type [WT]) and its mutation site (pMIR-SMAD1-mutanttype [MUT]) by referring to the instructions of pMIRREPORTTM (Ambion, Grand Island, NY, USA). The experimental steps were performed as reported previously. ${ }^{8}$ Then, $0.8 \mu \mathrm{g}$ WT or MUT reporter gene was transfected into HEK-293 cells, together with 50 nM microRNA mimics or microRNA-control. After transfection, the cells were incubated for 24 hours, and harvested to detect the luminescence of the reporter gene using the e-Dual-Glo luciferase assay (Promega, Madison, WI, USA). The luminescence of Renilla luciferase (Promega, Madison, WI, USA) was used to normalize the luminescence intensity of the target to be measured.

Statistical analysis. For RNA-sequencing, the fragments per kilobase of transcript per million fragments mapped (fpkm) value is determined by the calculation from the original data. For genes with fpkm greater than 2, we performed Student's t-test to generate a $p$-value for the difference between the 2 groups, followed by Benjamini-Hochberg correction. Subsequently, we used the Pearson correlation method for hierarchical clustering analysis and visualized the clustering results through genetic models. A volcano map was generated using the complete list of genes and is displayed in the image group. Kyoto encyclopedia of genes and Genomes (KEGG) pathway analysis and induction module analysis were performed using the reaction set data and were visualized using carcinogenic potency database (CPDB).

Statistical analysis. Using the Statistical Package for Social Sciences (SPSS) software version 17.0 (SPSS Inc., Chicago, IL, USA), the statistical differences between the experimental group and the control group were determined by double-tailed Student's t-test. All the experiments in this study were repeated 3 times, that is, the number of experimental group and control group were 3 . If the $p$-value $<0.05$, we will set a statistical difference between the 2 comparison groups.

Results. FBXW7 $\alpha$ knockdown alters the polarization of macrophages co-cultured with CRC. To determine whether FBXW7 $\alpha$ is important in the TAM phenotype, FBXW7 $\alpha$ levels in RAW264.7 macrophages were down-regulated using small interfering RNA siRNA, (Figure 1). Then the RAW264.7 macrophages were co-cultured with the mouse colon cancer cell line Colon-26 without direct cell-cell contact. Co-culture with Colon-26 can up-regulate the expression of M1 polarization markers of RAW264.7 macrophages, such as cyclooxygenase-2 (COX-2) mRNA, inducible nitric oxide synthase (iNOS) mRNA and IL6, IL12p40 and tumor necrosis factor- $\alpha$ (TNF- $\alpha$ ) production (Figure 1). The FBXW7 $\alpha$ knockdown macrophages co-cultured with Colon-26 can promote the expression of M1 markers, but the alternation can not be observed in macrophages without co-culture (Figure 1). These results indicate that FBXW7 $\alpha$ was important in blocking the TAM M1 polarization upon CRC co-culture.

Determination of global gene changes in $F B X W 7 \mathrm{\alpha}-$ knockdown macrophage cocultured with CRC. To investigate the molecular mechanism of FBXW7a in regulating the signal pathway in the TAM cocultured with CRC, we conducted RNA-seq to determine the macrophage gene expression. Heatmap cluster analysis indicated that FBXW7 $\alpha$ siRNA altered the transcription of numerous protein-coding and noncoding genes (Figure 2). Volcano plot of differential expressed genes showed that with the cut-off for fold change set at 2, a total of 648 genes were upregulated and 416 targets were downregulated (Figure 2). Table 1 lists the top 10 up-regulated genes and top 10 down- 
A

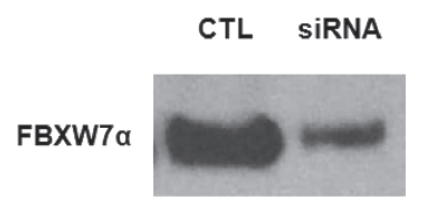

GAPDH

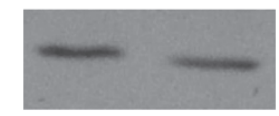

C

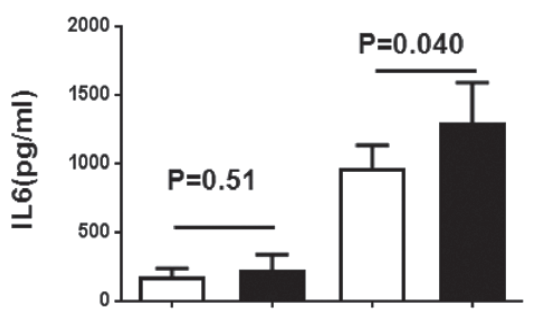

CTL SIRNA CTL SIRNA

Non-coculture $\overline{\text { coculture }}$
B
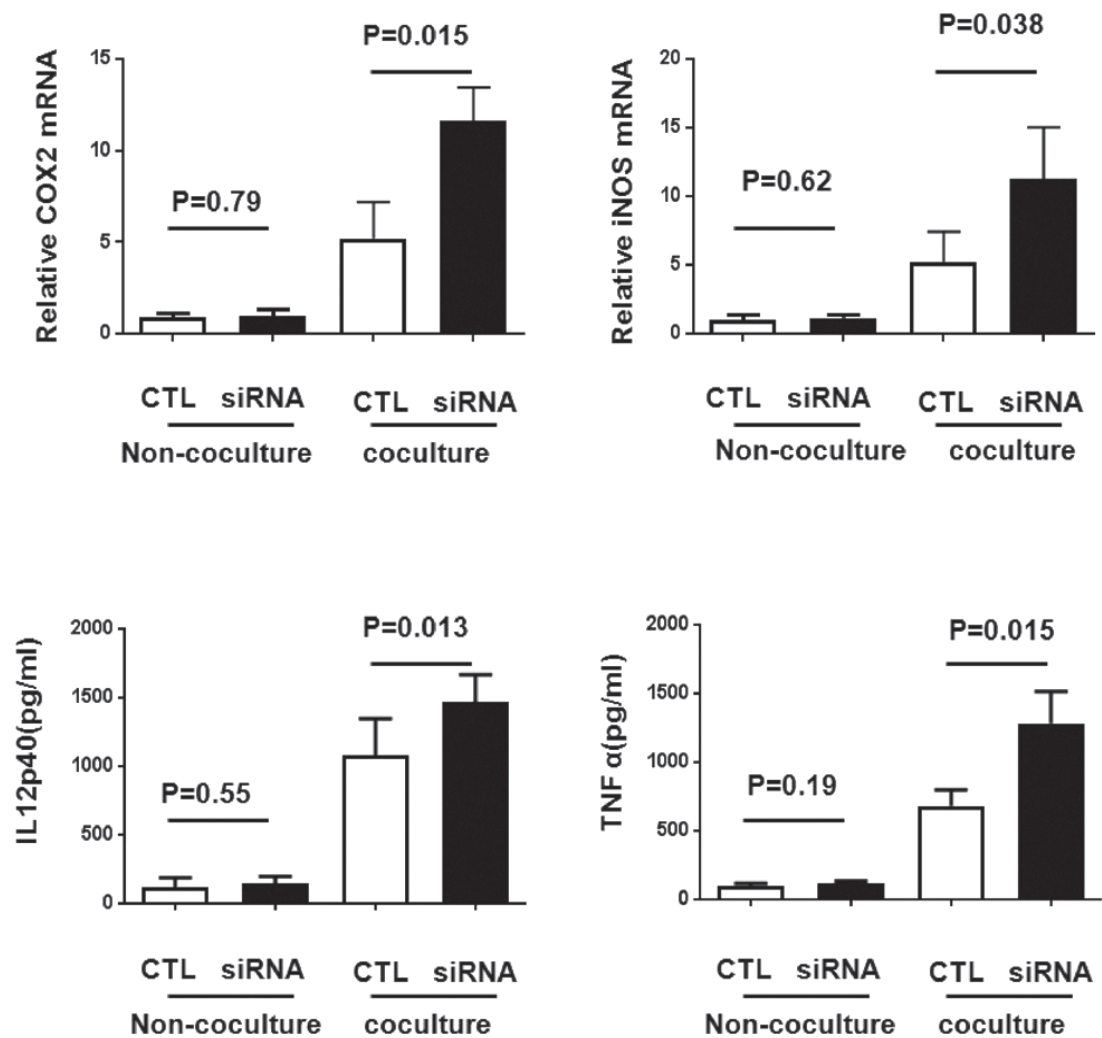

Figure 1 - Knockdown of FBXW7 $\alpha$ alters the phenotype of macrophage in response to colon cancer. A) Efficiency of interference plasmid transfection was examined in RAW264.7 macrophages. B-C) FBXW7 $\alpha$ siRNA plasmid or the control vector, were introduced into RAW264.7 for 36h, and then the cells were co-cultured with colon- 26 cells for $24 \mathrm{~h}$, B) In parallel, total RNA was isolated from co-cultured macrophage for quantitative reverse transcription-polymerase chain reaction (qRT-PCR) analysis of cyclooxigenase (COX)-2 and nitric oxide synthase 2 (NOS2); C) The cellfree supernatant was collected and analyzed by ELISA against IL6, IL12p40, and tumor necrosis factor- $\alpha$ (TNF $\alpha$ ). GAPDH - glyceraldehyde 3-phosphate dehydrogenase, iNOS - inducible nitric oxide synthase, CTL - control group

regulated genes. Among of the top 10 up-regulated genes, 6 were microRNAs. MiR-205 was the most significantly upregulated, with the $\log _{2}$ (fold change) at 9.041. Among of the top 10 down-regulated genes, Scn11a was the most significantly downregulated, with the $\log _{2}$ (fold change) at -11.4872 .

Validation of FBXW7a regulated genes identified by RNA-seq. We performed qRT-PCR for validation of FBXW7 $\alpha$ regulated genes identified by RNA-seq in Table 1. During qRT-PCR assay, 8 genes including 5 enhanced genes (miR-205, miR-148a, miR-144, miR-30d, and Lcn2) (Figure 2) and 3 reduced genes (RunX3, Tns1, and Cnnm1) (Figure 2D), showed expression patterns similar to those observed in an integrated analysis. Among these, miR-205 exhibited the most significant upregulation, which was consistent with the RNA-seq results. Scn11a also showed the most significant downregulation upon FBXW7 $\alpha$ siRNA treatment.

MiR-205 is required in the regulation of TAM polarization by $F B X W 7 \alpha$. To test weather miR-205 was required in the polarization alternation triggered by FBXW7 $\alpha$ knockdown, the Colon- 26 co-cultured or non-co-cultured macropahges were transfected with miR-205 inhibitors or a negative control, together with FBXW7 $\alpha$ or not, for 24 hours, and then the IL6, IL12p40 and TNF $\alpha$ production were measured. In the non-co-cultured macrophages, neither the microRNA205 inhibitor nor the FBXW7 $\alpha$ siRNA could significantly alter the level of these inflammatory factor. In the co-cultured macrophages, FBXW7 $\alpha$ siRNA enhanced IL6, IL12p40 and TNFa were significantly reversed by $m i R-205$ inhibitor, whereas little effect was observed in negative control. MiR-205 treatment without FBXW7 $\alpha$ siRNA could down-regulated the 
A

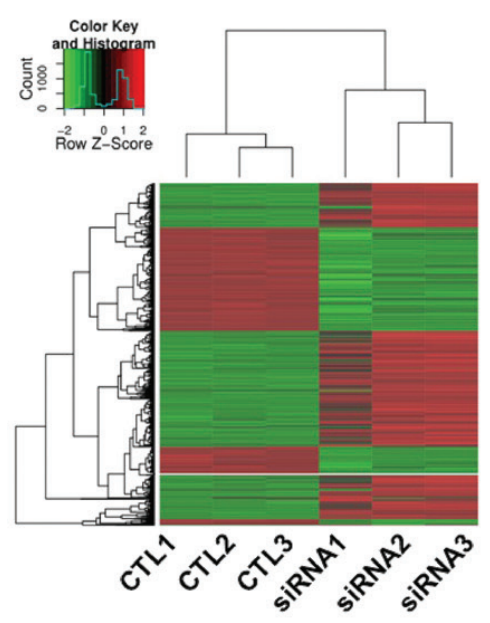

C
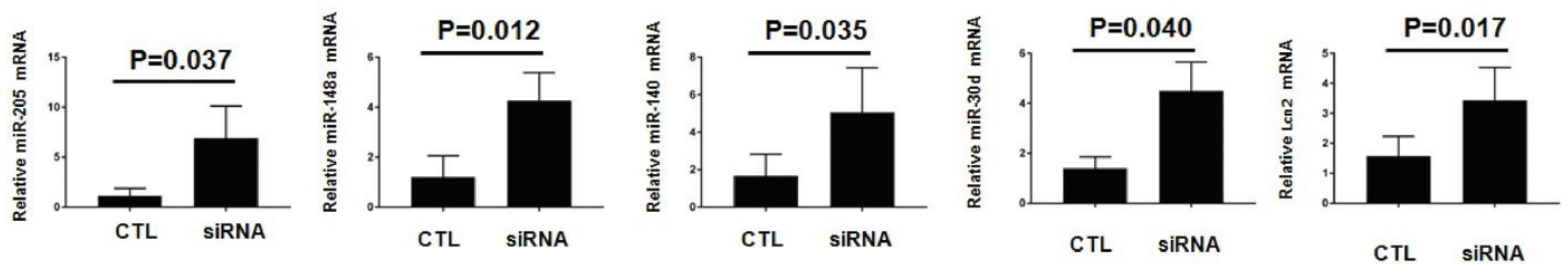

D
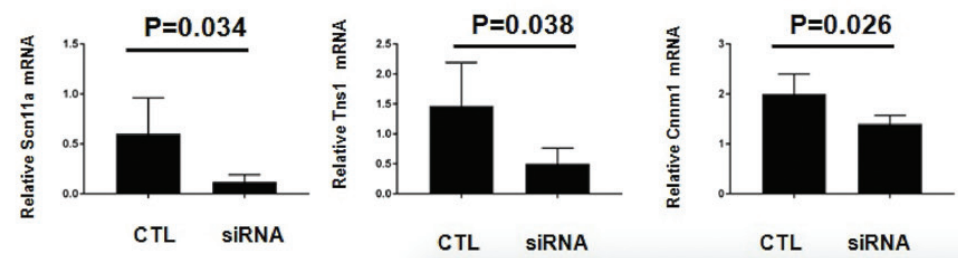

Figure 2 - Genome-wide sequencing together with quantitative reverse transcription-polymerase chain reaction (qRT-PCR) analysis identifies FBXW7 $\alpha$ regulated genes of macrophage in response to colon cancer. A) the RNA samples prepared in Figure 1B were used to perform the RNA-seq experiment. Heatmap analysis displaying the dis-regulated targets in FBXW7 $\alpha$ siRNA transfected macrophage, comparing with the control group. B) Volcano plot displaying the number of genes with significant differences. C) qRT-PCR analysis of validate five up-regulated targets identified by RNA-seq. D) qRT-PCR analysis of validate three down-regulated targets identified by RNA-seq. CTL - control group, siRNA: FBXW7 $\alpha$ - interference plasmid transfection group.

inflammatory factor productions slightly, but the difference was not statistically significant (Figure 3). These results indicated that $m i R-205$ is required in the polarization alternation of FBXW7 $\alpha$ knockdown macrophage co-cultured with CRC.

SMAD1 is a target of miR-205. We next explored the molecular mechanism of microRNA-205 regulating macrophage polarization. Using the Targetscan website (http://www.targetscan.org), we identified a potential target protein, SMAD1, for microRNA-205, which is closely related to macrophage polarization (Figure 4A). In order to directly determine whether or not microRNA-205 binds to the SMAD1 3'UTR, we performed in vitro luciferase reporter experiment. As shown in Figure 4B, we observed a significant decrease in luciferase activity of cells transfected with 
Table 1 - Top 10 up-regulated genes and top 10 down-regulated genes from the RNA-seq results in FBXW7a knockdown macrophage cocultured with CRC.

\begin{tabular}{lccc}
\hline Gene name & $\log _{2}($ fold change $)$ & $P$-value & Q-value \\
\hline Up-regulated & & & \\
Mir205 & 9.041 & 0.000229 & 0.002748 \\
Mir451 & 8.59988 & 0.000352 & 0.003782 \\
Mir148a & 8.08949 & 0.000848 & 0.008609 \\
Mirlet7b & 7.91503 & 0.001402 & 0.013246 \\
Mir30d & 6.74795 & $2.92 \mathrm{E}-11$ & $1.41 \mathrm{E}-09$ \\
Mir185 & 6.49331 & 0.000756 & 0.007806 \\
Mmp9 & 6.4013 & 0.000288 & 0.003364 \\
Lcn2 & 6.22779 & 0.000721 & 0.007506 \\
Mir140 & 6.1842 & $7.42 \mathrm{E}-07$ & $1.67 \mathrm{E}-05$ \\
iNOS & 6.15058 & $7.37 \mathrm{E}-10$ & $2.78 \mathrm{E}-08$ \\
Down-regulated & & & \\
Scn11a & -11.4872 & $1.36 \mathrm{E}-05$ & 0.000263 \\
Cnnm1 & -9.73811 & $6.58 \mathrm{E}-09$ & $2.09 \mathrm{E}-07$ \\
Ranbp3l & -9.22856 & 0.000544 & 0.006329 \\
Prss35 & -9.09956 & 0.000342 & 0.003781 \\
Tns1 & -8.54023 & $1.94 \mathrm{E}-09$ & $6.74 \mathrm{E}-08$ \\
Rorc & -8.24786 & $5.94 \mathrm{E}-06$ & 0.00011 \\
Scel & -7.7752 & $1.72 \mathrm{E}-05$ & 0.000225 \\
Igf2bp3 & -7.53597 & $8.22 \mathrm{E}-15$ & $5.81 \mathrm{E}-13$ \\
H2-Eb1 & -7.53352 & $3.59 \mathrm{E}-05$ & 0.000546 \\
Chst1 & -7.32653 & $4.66 \mathrm{E}-05$ & 0.000673 \\
\hline
\end{tabular}

a mimic of $m i R-205$ and a luciferase reporter vector. Conversely, luciferase activity was not observed in cells transfected with the 3'UTR-NC or 3'MUT. The above data indicate that SMAD1 could be directly targeted by microRNA-205.

The FBXW7a/miR-205 axis regulates TAM polarization by affecting SMAD1 expression. Next, the mRNA and protein expression of SMAD1 in Colon-26 co-cultured macrophages was measured. First, we found that FBXW7 $\alpha$ siRNA effect was sustained throughout the duration of miR-205 treatment. Meanwhile, FBXW7 $\alpha$ siRNA significantly reduced the mRNA and protein expression of SMAD1, whereas treatment with the miR-205 inhibitor could block this function (Figure 4C-4D). Further, SMAD1 overexpression could reverse the FBXW7 $\alpha$ siRNA enhanced IL6, IL12p40, and TNFa production significantly (Figure 4E-4G). Taken together, our results demonstrate that the FBXW7 $\alpha / m i R-205$ axis regulates M1/M2 polarization by affecting SMAD1 expression in macrophages cocultured with CRC.

Discussion. Chronic inflammation is closely related to the onset of colon cancer.' Inflammatory factors are mainly derived from immune cells, and studies have shown that regulation of inflammation in tumors is a double-edged sword. ${ }^{10}$ Tumor-polarized M1/M2 macrophage polarization in tumor progression
A
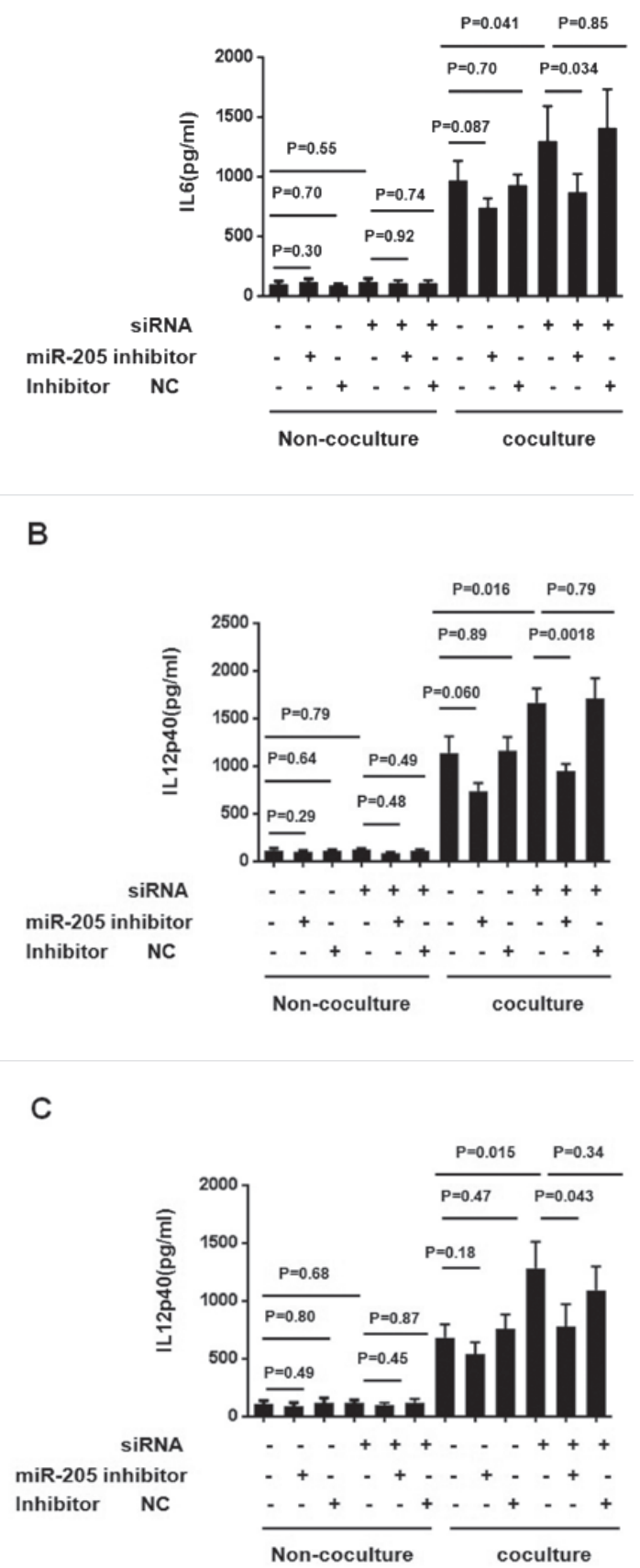

Figure 3 - MiR-205 is required in the regulation of tumor-associated macrophages (TAM) polarization by FBXW7 $\alpha$. A-C) FBXW7 $\alpha$ siRNA plasmid or control vector, together with $m i R-205$ inhibitor or inhibitor negative control (inhibitor NC), were introduced into RAW264.7 for 36-hours, and then the cells were co-cultured with Colon-26 cells for 24-hours. The cell-free supernatant was collected and analyzed by ELISA against A) IL6, B) IL12p40, and C)TNF $\alpha$. siRNA: FBXW7 $\alpha$ interference plasmid transfection group. $\mathrm{NC}$ - negative control group. 
A

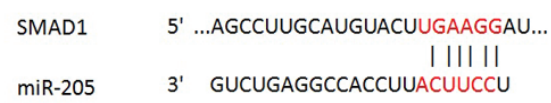

C

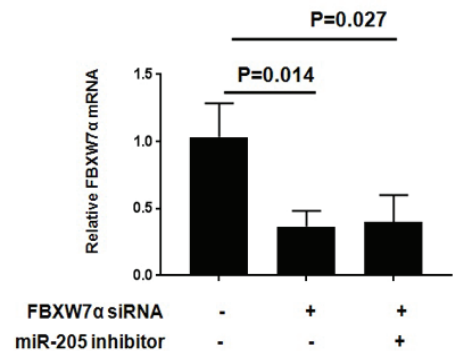

E

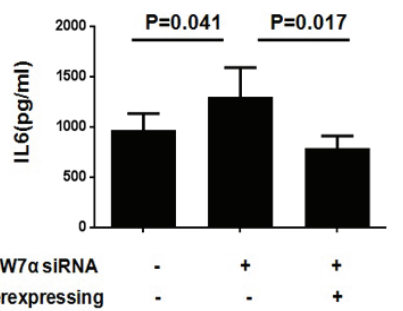

F

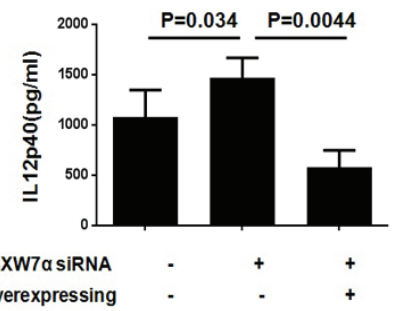

B

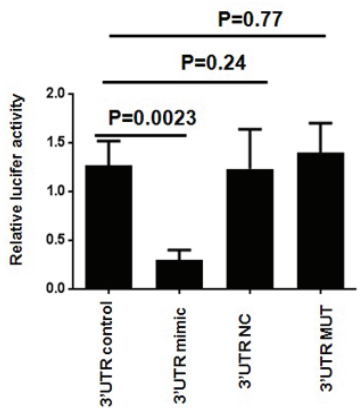

D

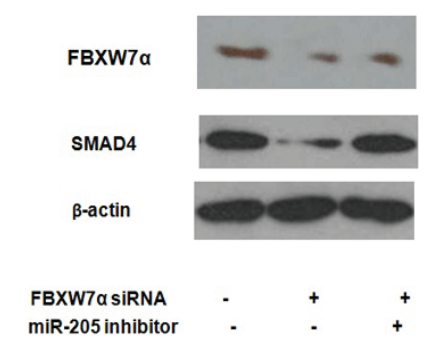

G

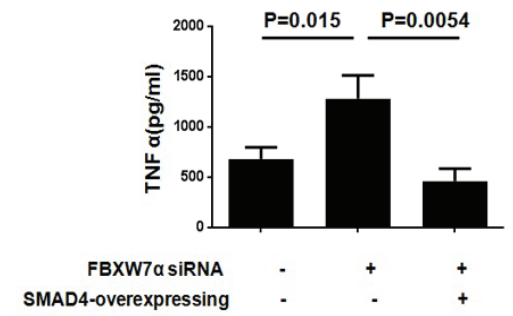

Figure 4 - FBXW7 $\alpha / m i R-205$ axis regulate tumor-associated macrophages (TAM) polarization through affecting SMAD1 expression. A-B) SMAD1 is a target of miR-205. A) The region of the human SMAD1 3'UTR predicted to be targeted by miR-205. B) HEK-293T cells were transiently cotransfected with luciferase reporter vectors, and either miR-205 mimics or negative control. Luciferase activities were normalized to the activity of Renilla luciferase. (C-D) FBXW7 $\alpha$ siRNA plasmid or control vector, together with $m i R-205$ inhibitor or inhibitor negative control (inhibitor NC), were introduced into RAW264.7 for 36-hours, and then the cells were cocultured with Colon-26 cells for 24-hours. C) The mRNA level was determined by quantitative reverse transcription-polymerase chain reaction (qRT-PCR) assay; and D) protein level was determined by western-blotting assay. (E-G FBXW7 $\alpha$ siRNA plasmid or control vector, together with SMAD1 overexpressing plasmid or NC, were introduced into RAW264.7 for 36-hours, and then the cells were cocultured with Colon-26 cells for 24-hours. The cell-free supernatant was collected and analyzed by ELISA against E) IL6, (F) IL12p40 and G) tumor necrosis factor- $\alpha$ (TNF $\alpha$ ).

may represent a link between tumor development, pro-inflammatory responses and tumor progression. ${ }^{11}$ Here, we report our findings that FBXW7 $\alpha$ plays an negative role in TAM M1 polarization, and that FBXW7 $\alpha$ siRNA could help increase the expression of M1 markers, including the secretion of TNF- $\alpha$, IL-12, and IL-6, and COX2 and NOS2 expression in the cytoplasm. Altered TAM polarization was reported to be highly related with the cancer cell division, growth, and differentiation. ${ }^{12}$ This might be the main reason why FBXW7 $\alpha$ has been characterized as a general tumor suppressor. In order to explore the regulatory mechanism of FBXW7 $\alpha$ in TAM polarization, we tested colon cancer co-cultured RAW264.7 cells after FBXW7 $\alpha$ silencing for genome-wide differential genes determination.

Ribonucleic acid-sequencing assay, together with qRT-PCR validation identified several targets with the most significant differential expression between FBXW7 $\alpha$ silenced and control group. Among them, $m i R-205$ was the most significantly overexpressed. 
MiR-205 has been reported to regulate tumor development by targeting multiple genes. Wang et $\mathrm{al},{ }^{13}$ demonstrated that miR-205 inhibits the development of renal cell carcinoma through regulating the PTEN/AKT pathway. Ji et al, ${ }^{14}$ reported that $m i R-205$ functions by targeting Yap1 in glioma. Further, Zeng et $\mathrm{al}^{8}{ }^{8}$ found that miR-205 could repress SMAD4 expression in A549 cell lines, which was similar to our results. SMAD family signaling has been reported to regulate macrophage proliferation and activities, thus inhibiting inflammation. ${ }^{15}$ Our work describes the signaling pathway from the upstream element FBXW7 $\alpha$ to TGF- $\alpha /$ SMAD1, which was mediated by miR-205. This pathway clearly plays an important role in regulating the TAM M1/M2 polarization switch and might be involved in tumor development. Notably, besides miR-205, several microRNAs are induced by FBXW7 $\alpha$ silencing, including miR-148a, miR-140, and miR-30d. ${ }^{16-18}$ All of these targets are highly related with TAM functions. This suggests that the FBXW7 $\alpha$ function in tumor development is complex and needs further detailed and comprehensive study.

In conclusion, our study proved that the FBXW7 $\alpha / m i R-205$ axis plays an important role in TAM polarization and could therefore block CRC development. The limitation of the article is that we did not verify our findings in animal experiments or in clinical specimens. Nevertheless, our results provide a new possibility for a follow-up study of colon cancer treatment, that is, blocking the growth of colon cancer by regulating the macrophage FBXW7 $\alpha / m i R-205$ axis in the tumor microenvironment.

Acknowledgment. The authors gratefully acknowledge Editage (https://www.editage.com/) for English language editing.

\section{References}

1. Torre LA, Bray F, Siegel RL, Ferlay J, Lortet-Tieulent J, Jemal A. Global cancer statistics, 2012. CA Cancer J Clin 2015; 65: 87-108.

2. Isidro RA, Appleyard CB. Colonic macrophage polarization in homeostasis, inflammation, and cancer. Am J Physiol Gastrointest Liver Physiol 2016; 311: G59-G73.

3. Peng L, Zhang H, Hao Y, Xu F, Yang J, Zhang R, et al. Reprogramming macrophage orientation by microRNA $146 \mathrm{~b}$ targeting transcription factor IRF5. EBioMedicine 2016; 14: $83-96$.
4. Yeh CH, Bellon M, Nicot C. FBXW7: a critical tumor suppressor of human cancers. Mol Cancer 2018; 17: 115.

5. Kothari N, Teer JK, Abbott AM, Srikumar T, Zhang Y, Yoder SJ, et al. Increased incidence of FBXW7 and POLE proofreading domain mutations in young adult colorectal cancers. Cancer 2016; 122: 2828-2835.

6. Xie T, Cho YB, Wang K, Huang D, Hong HK, Choi YL, et al. Patterns of somatic alterations between matched primary and metastatic colorectal tumors characterized by whole-genome sequencing. Genomics 2014; 104: 234-241.

7. Mlecnik B, Bindea G, Kirilovsky A, Angell HK, Obenauf AC, Tosolini $\mathrm{M}$, et al. The tumor microenvironment and Immunoscore are critical determinants of dissemination to distant metastasis. Sci Transl Med 2016; 8: 327ra26.

8. Zeng Y, Zhu J, Shen D, Qin H, Lei Z, Li W, et al. Repression of Smad4 by miR-205 moderates TGF- $\beta$-induced epithelialmesenchymal transition in A549 cell lines. Int J Oncol 2016; 49: 700-708.

9. Saxena A, Fayad R, Kaur K, Truman S, Greer J, Carson JA, et al. Dietary selenium protects adiponectin knockout mice against chronic inflammation induced colon cancer. Cancer Biol Ther 2017; 18: 257-267.

10. O’Malley G, Heijltjes M, Houston AM, Rani S, Ritter T, Egan LJ, et al. Mesenchymal stromal cells (MSCs) and colorectal cancer: a troublesome twosome for the anti-tumour immune response? Oncotarget 2016; 7: 60752-60774.

11. Mantovani A, Marchesi F, Malesci A, Laghi L, Allavena P. Tumour-associated macrophages as treatment targets in oncology. Nat Rev Clin Oncol 2017; 14: 399-416.

12. Petty AJ, Yang Y. Tumor-associated macrophages: implications in cancer immunotherapy. Immunotherapy 2017; 9: 289-302.

13. Wang H, Chen B, Duan B, Zheng J, Wu X. miR-205 suppresses cell proliferation, invasion, and metastasis via regulation of the PTEN/AKT pathway in renal cell carcinoma. Mol Med Rep 2016; 14: 3343-3349.

14. Ji T, Zhang X, Li W. microRNA-205 acts as a tumor suppressor and directly targets YAP1 in glioma. Mol Med Rep 2017; 16: 1431-1438.

15. Means AL, Freeman TJ, Zhu J, Woodbury LG, MarincolaSmith P, Wu C, et al. Epithelial smad4 deletion up-regulates inflammation and promotes inflammation-associated cancer. Cell Mol Gastroenterol Hepatol 2018; 6: 257-276.

16. Shi L, Xi J, Xu X, Peng B, Zhang B. MiR-148a suppressed cell invasion and migration via targeting WNT10b and modulating $\beta$-catenin signaling in cisplatin-resistant colorectal cancer cells. Biomed Pharmacother 2019; 109: 902-909.

17. Flamini V, Jiang WG, Cui Y. Therapeutic role of MiR-140-5p for the treatment of non-small cell lung cancer. Anticancer Res 2017; 37: 4319-4327.

18. Melman YF, Shah R, Danielson K, Xiao J, Simonson B, Barth A, et al. Circulating microRNA-30d is associated with response to cardiac resynchronization therapy in heart failure and regulates cardiomyocyte apoptosis: a translational pilot study. Circulation 2015; 131: 2202-2216. 